\title{
Default Bayesian Analysis for Multivariate Generalized CAR Models
}

\author{
Sarat C. Dass, Chae Young Lim and Tapabrata Maiti* \\ Department of Statistics \& Probability \\ Michigan State University, East Lansing, MI 48824 \\ Email: \{sdass,lim,maiti\}@stt.msu.edu
}

\begin{abstract}
In recent years, multivariate spatial models have been proven to be an effective tool for analyzing spatially related multidimensional data arising from a common underlying spatial process. Currently, the Bayesian analysis is perhaps the only solution available in this framework where prior selection plays an important role in the inference. The present article contributes towards the development of Bayesian inferential methodology for multivariate generalized linear mixed models, in particular, multivariate conditional autoregressive (CAR) models. The two main contributions of this article are the development of a shrinkage-type default prior for the covariance matrices and innovative computational techniques for the Gibbs sampling implementation. The default prior elicitation is non-informative but results in a proper posterior on the related parameter spaces. This elicitation not only provides robust inference (with respect to prior choice), but also provides improved estimation. In the computational step, we have developed a transformation of the parameters that avoids sampling from restricted domains, thus providing more stability and efficiency in the Gibbs implementation. The methodology has been extended to the case of missing responses in the multi-dimensional setup. Both simulations and real examples are provided to validate and illustrate the proposed methodology.
\end{abstract}

Keywords: Conditional autoregressive models, Default Bayesian analysis, Generalized linear mixed models, Gibbs sampling, Spectral decomposition

*Authors' names are in alphabetical order. 


\section{Introduction}

Many spatial problems, particularly those concerning environmental, health and socio-economic variables, are inherently multivariate, meaning that two or more such variables are recorded at each spatial location simultaneously. With rapid enhancements in geographic information systems (GIS) technology that enables us to analyze and display such data at varying spatial resolutions, multivariate spatial analysis is becoming more relevant and popular. Sain and Cressie (2007) viewed the developments of spatial analysis in two main categories: models for geostatistical data (that is, the indices of data points belong in a continuous set) and models for lattice data (data with indices in a discrete or countable set), while specifically mentioning that the latter is not as developed.

The issue of "health disparity" is central to the distribution of federal and state aid based on socio-economic indicators. Health disparity studies analyze how health status of individuals vary across various socio-economic groups and spatial locations, in particular in relation to a specific disease. Multiple response variables are available as indicators of health status, and as a result, models for multivariate spatial lattice data are an indispensable tool for analyzing health disparity data. Recently, Greco and Trivisano (2009), Zhang et al. (2009), Jin et al (2007), Sain and Cressie (2007), Jin et al. (2005), Gelfand and Vounatsou (2003), Carlin and Banerjee (2003) and Kim et al. (2001) explored multivariate spatial models for lattice data, adopting the Bayesian framework as the natural inferential approach. The only exception, Sain (2009) developed the maximum likelihood estimation procedure for a special case, the multivariate gaussian conditional autoregressive (CAR) model of Sain and Cressie (2007).

Although the Bayesian inferential framework is a natural choice for spatial lattice data, one obvious obstacle is the choice of prior distribution for the model parameters. All previous works mentioned above are based on standard subjective, and at best, vague priors to account for the lack of subjective knowledge. Subjective specification of priors have the obvious drawback of introducing bias in the estimation procedure, the extent of which may not be easy to gauge in applications. A default, or non-informative prior, is therefore preferable for the Bayesian approach. By default prior, we mean the type of reference prior considered in Natarajan and Kass (2000). However, in the case of hierarchical generalized linear models, putting non-informative priors (i.e., improper priors) on covariance parameters may result in the posterior being improper. This led Natarajan and Kass (2000) to develop default priors in the context of generalized linear mixed models (GLMM) for which the posterior can be established to be proper.

A generalized linear mixed model (GLMM) with a multivariate Gaussian CAR model can be viewed as a special case of a general random effects model with specific restrictions on the 
structure of the covariance matrix. The Natarajan-Kass prior neither takes into account the special structure of the covariance matrix nor considers the dimension reduction capabilities of a CAR model in the spatial case. In fact, some simple modifications greatly reduce the number of parameters while maintaining flexibility in modeling (Sain and Cressie, 2007).

In this paper, we develop a new default prior for the multivariate CAR models. The simulation analysis using subjective and vague priors indicates significant prior sensitivity compared to the proposed prior, which justifies the use of the latter in real applications. Our Bayesian computational scheme is different from previous approaches. We adopt a new parametrization of the multivariate CAR model based on the Cholesky and spectral decompositions of matrices. The enormous advantage gained through this re-parametrization is the removal of constraints on the parameter space induced by the positive definiteness requirement on the inverse covariance matrix.

The CAR model we consider in this paper can be considered as an extension of the multivariate CAR model in Gelfand and Vounatsou (2003) and Carlin and Banerjee (2003) and as a special case of Sain and Cressie (2007). This model is also similar to the model in Jin et al (2007) or the model in Greco and Trivisano (2009). Both papers discuss prior choices for the parameters but they are somewhat different from ours. The priors considered here are theoretically and methodologically justified from the prospective of Jeffrey's prior and guaranteed posterior propriety. Among other significant differences, this paper contributes towards Bayesian computations which efficiently implement the multivariate CAR models.

This work is motivated from authors' recent project on joint mapping of cancer mortality data and a socio-economic variable (or an environmental variable) for all counties in the state of Michigan. In the project, we are interested in addressing whether health and socio-economic disparities (or environmental indicators) are spatially correlated and whether accounting of such dependence improves estimation of relative risks of cancer. It is often the case that cancer mortality or incidence data is only partially available due to confidentiality. Also, environmental data are available only in the area where a measurement station is located. Thus we incorporate partially observed or missing data case in developing a default prior. The Bayesian inferential procedure is illustrated through simulation and an application based on cancer mortality data from Surveillance, Epidemiology, and End Results (SEER) Program (www.seer.cancer.gov) for the state of Michigan. Detail description of data is given in 5 .

The rest of the article is organized as follows: Section 2 introduces the multivariate CAR model in the spatial context. Section 3 discusses the motivation for developing the proposed default prior by taking the spatial information into account. The resulting posterior distribution is shown to be proper in this section for both complete and missing data cases. The Gibbs steps are outlined in Section 4 whereas Section 5 gives the numerical findings 
for both simulated and real data. This is followed by a brief discussion summarizing our findings in Section 6 and the Appendix.

\section{Multivariate Generalized Linear Mixed Models}

In what follows, we assume that there are $n$ distinct sites on a spatial domain where observations on $p$ variables are recorded. Let the multivariate data consist of the $p$-dimensional random vector $\mathbf{y}_{j} \equiv\left(y_{1 j}, y_{2 j}, \cdots, y_{p j}\right)^{\prime}$ for the $j$-th site, for $j=1,2, \cdots, n$. Corresponding to the response $y_{i j}$, denote by $x_{i j}=\left(x_{i j 1}, x_{i j 2}, x_{i j q_{i}}\right)^{\prime}$ to be the $q_{i} \times 1$ vector of explanatory variables. The following two stage hierarchical model is considered for the distribution of the $n p \times 1$ vector of all observables $\mathbf{y} \equiv\left(\mathbf{y}_{1}^{\prime}, \mathbf{y}_{2}^{\prime}, \cdots, \mathbf{y}_{n}^{\prime}\right)^{\prime}$ : In the first stage of the hierarchy, the variables $y_{i j}$ are independent with density

$$
f_{i j}\left(y \mid \eta_{i j}\right)=C(y) \exp \left\{\eta_{i j} y-h_{i}\left(\eta_{i j}\right)\right\}
$$

where $f_{i j}$ belongs to an exponential family of densities with canonical parameter $\eta_{i j}$, and $h_{i}\left(\eta_{i j}\right)$ is the normalizing constant satisfying

$$
\exp \left\{h_{i}\left(\eta_{i j}\right)\right\}=\int C(y) \exp \left\{\eta_{i j} y\right\} d y
$$

It can be shown (see, for example, McCullagh and Nelder (1989)) that $h_{i}(\cdot)$ is a differentiable function with inverse $h_{i}^{-1}$. In the second stage, the canonical parameter $\eta_{i j}$ is related to $x_{i j}$ via a link function in the usual generalized linear model set-up,

$$
\eta_{i j}=x_{i j}^{\prime} \beta_{i}+\epsilon_{i j}
$$

where $\beta_{i}$ is a $q_{i} \times 1$ vector of regression coefficients and $\epsilon_{i j}$ are error random variables. The hierarchical specification is completed by eliciting the distribution for the error component $\epsilon_{i j}$ s, namely, $\boldsymbol{\epsilon} \sim N_{n p}(0, \boldsymbol{D})$ where $\boldsymbol{\epsilon}_{j} \equiv\left(\epsilon_{1 j}, \epsilon_{2 j}, \cdots, \epsilon_{p j}\right)^{\prime}$ is the $p \times 1$ error vector at the $j$-th spatial site, $\boldsymbol{\epsilon} \equiv\left(\boldsymbol{\epsilon}_{1}^{\prime}, \boldsymbol{\epsilon}_{2}^{\prime}, \cdots, \boldsymbol{\epsilon}_{n}^{\prime}\right)^{\prime}$ is the $n p \times 1$ vector of all the error variables and $\boldsymbol{D}$ is the covariance matrix (of dimension $n p \times n p$ ) of $\boldsymbol{\epsilon}$. Such models based on an unstructured $\boldsymbol{D}$ are called a random-effects GLMs or generalized linear mixed models (GLMMs), and has been the subject of many theoretical as well as practical studies in recent years.

\subsection{Multivariate Gaussian CAR}

In the spatial context, the distribution of $\boldsymbol{\epsilon}$, and hence $\boldsymbol{D}$, can be given a more concrete structure based on neighboring dependencies. Following Mardia (1988), we define the multivariate Gaussian CAR model as follows: For some $p \times p$ matrices $\boldsymbol{\Gamma}_{j}$ and $\boldsymbol{\Lambda}_{j k}$, suppose that 
the vector $\boldsymbol{\epsilon}_{j}$ is $p$-variate Gaussian with

$$
E\left(\boldsymbol{\epsilon}_{j} \mid \boldsymbol{\epsilon}_{-j}\right)=\sum_{k \in N_{j}} \boldsymbol{\Lambda}_{j k} \boldsymbol{\epsilon}_{k}, \quad \text { and } \quad \operatorname{Var}\left(\boldsymbol{\epsilon}_{j} \mid \boldsymbol{\epsilon}_{-j}\right)=\boldsymbol{\Gamma}_{j}, \quad \text { for } j=1,2, \cdots, n,
$$

where $\boldsymbol{\epsilon}_{-j} \equiv\left\{\boldsymbol{\epsilon}_{k}: k \in N_{j}\right\}$ denotes the rest of the $\boldsymbol{\epsilon}$ at all locations $k$ in the neighborhood $N_{j}$. The existence of the joint distribution for $\epsilon$ is guaranteed by the symmetry and positive definiteness of $\boldsymbol{D}$ which, respectively, translates to

$$
\Lambda_{j k} \Gamma_{k}=\Gamma_{j} \Lambda_{k j}^{\prime}
$$

for all pairs $(j, k)$, and $\operatorname{Block}\left(-\boldsymbol{\Gamma}_{j}^{-1} \boldsymbol{\Lambda}_{j k}\right)$ is positive definite with $\boldsymbol{\Lambda}_{j j}=-\mathbf{I}$. Then, from Mardia (1988), $\boldsymbol{\epsilon}$ is $N_{n p}(\mathbf{0}, \boldsymbol{D})$ with

$$
\boldsymbol{D}=\left\{\operatorname{Block}\left(-\boldsymbol{\Gamma}_{j}^{-1} \boldsymbol{\Lambda}_{j k}\right)\right\}^{-1}
$$

We introduce some more notation here relevant to the spatial context. The weight matrix $\boldsymbol{W}=\left(\left(w_{j k}\right)\right)($ of dimension $n \times n)$ consists of entries

$$
w_{j k}= \begin{cases}1 & \text { if } j \text { and } k \text { are neighbors, and } \\ 0, & \text { otherwise }\end{cases}
$$

with $w_{j j} \equiv 0$ for $j, k=1,2, \cdots, n$. For each site $j$, define $w_{j+} \equiv \sum_{k \in N_{j}} w_{j k}$ to be the sum that represents the total number of neighbors of site $j$, and $\boldsymbol{M}$ to be the $n \times n$ diagonal matrix

$$
\boldsymbol{M}=\operatorname{diag}\left(w_{1+}, w_{2+}, \cdots, w_{n+}\right) .
$$

It is convenient both computationally as well as for practical analysis to represent all the $\boldsymbol{\Gamma}_{j}$ and $\boldsymbol{\Lambda}_{j k}$ in terms of two common $p \times p$ matrices $\boldsymbol{\Gamma}$ and $\boldsymbol{H}$, respectively. The first stage of parametrization is to take

$$
\boldsymbol{\Gamma}_{j}=\frac{\boldsymbol{\Gamma}}{w_{j+}} \quad \text { and } \quad \boldsymbol{\Lambda}_{j k}=\frac{w_{j k}}{w_{j+}} \cdot \boldsymbol{H} .
$$

The first part of equation (8) entails a common covariance matrix $\boldsymbol{\Gamma}$ for all sites $j$, rescaled by the weight factor $w_{j+}^{-1}$. It follows that sites with a larger number of neighbors will have lower variability, which is reasonable to expect. The second part of equation (8) entails a common set of regression coefficients $\boldsymbol{H}$ rescaled by the inverse of the number of neighbors $w_{j+}$. The matrix $\boldsymbol{\Gamma}$ must be positive definite since $\boldsymbol{\Gamma}_{j}$ represents the covariance matrix of $\boldsymbol{\epsilon}_{j}$ given $\boldsymbol{\epsilon}_{-j}$. Substituting (8) in (4), the symmetry requirement of (4) is equivalent to

$$
\boldsymbol{H} \Gamma=\Gamma \boldsymbol{H}^{\prime}
$$


or, in other words, $\boldsymbol{F} \equiv \boldsymbol{\Gamma}^{-1 / 2} \boldsymbol{H} \boldsymbol{\Gamma}^{1 / 2}$ should be symmetric, where $\boldsymbol{\Gamma}^{1 / 2}$ is the (unique) square root matrix of $\boldsymbol{\Gamma}$ and $\boldsymbol{\Gamma}^{-1 / 2}$ is its inverse.

With the above re-parametrization, the distribution of $\boldsymbol{\epsilon}$ is multivariate normal with mean 0 and covariance matrix $\boldsymbol{D}$, which is now a function of $\boldsymbol{\Gamma}$ and $\boldsymbol{F}$ only. In fact, the inverse of $\boldsymbol{D}$ has the expression

$$
\boldsymbol{D}^{-1}=\left(\boldsymbol{I}_{n} \otimes \boldsymbol{\Gamma}^{-1 / 2}\right)\left(\boldsymbol{M} \otimes \boldsymbol{I}_{p}-\boldsymbol{W} \otimes \boldsymbol{F}\right)\left(\boldsymbol{I}_{n} \otimes \boldsymbol{\Gamma}^{-1 / 2}\right)
$$

where $A \otimes B$ represents the Kronecker product of two matrices $A$ and $B$. It can be easily shown that positive definiteness of $\boldsymbol{D}^{-1}$ is ensured by restricting eigenvalues of $\boldsymbol{F}=$ $\boldsymbol{\Gamma}^{-1 / 2} \boldsymbol{H} \boldsymbol{\Gamma}^{1 / 2}$ to be in $(-1,1)$ (see the Appendix for a proof).

The concatenated vector of all $\eta_{i j}$ s at the $j$-th spatial location is denoted by $\boldsymbol{\eta}_{j} \equiv$ $\left(\eta_{1 j}, \eta_{2 j}, \cdots, \eta_{p j}\right)^{\prime}$. Further, $\boldsymbol{\eta} \equiv\left(\boldsymbol{\eta}_{1}^{\prime}, \boldsymbol{\eta}_{2}^{\prime}, \cdots, \boldsymbol{\eta}_{n}^{\prime}\right)^{\prime}$ represents the $n p \times 1$ vector of all $\eta_{i j}$ variables. Since $\boldsymbol{\epsilon} \sim N_{n p}(0, \boldsymbol{D})$, it follows that

$$
\boldsymbol{\eta} \sim N_{n p}(\boldsymbol{X} \boldsymbol{\beta}, \boldsymbol{D})
$$

where $\boldsymbol{\beta}=\left(\beta_{1}^{\prime}, \beta_{2}^{\prime}, \cdots, \beta_{p}^{\prime}\right)^{\prime}$ is the $q \times 1$ concatenated vector of all regression coefficients with $q=\sum_{i=1}^{p} q_{i}$, and $\boldsymbol{X}$ is the $p n \times q$ design matrix given by

$$
\boldsymbol{X}=\left(\begin{array}{c}
X_{1} \\
X_{2} \\
\vdots \\
X_{n}
\end{array}\right)_{(n p \times q)}, \quad \text { with } \quad \boldsymbol{X}_{j}=\left(\begin{array}{cccc}
x_{1 j}^{\prime} & 0 & \cdots & 0 \\
0 & x_{2 j}^{\prime} & \cdots & 0 \\
\vdots & \vdots & \ddots & \vdots \\
0 & 0 & \cdots & x_{p j}^{\prime}
\end{array}\right)_{(p \times q)}
$$

denoting the design matrix at the $j$-th spatial location for $j=1,2, \cdots, n$.

\subsection{Handling Partial and Missing Observations}

In some applications, part of the $\boldsymbol{y}$ observations can be either missing or partially observed, thus requiring the development of spatial hierarchical GLMMs for partially observed data structures. The sets $\mathcal{M}, \mathcal{P}$ and $\mathcal{C}$ represent all pairs $(i, j)$ where $y_{i j}$ is either missing, partially observed or completely observed, respectively. Given $\boldsymbol{\eta}$, the conditional likelihood contribution corresponding to the partially observed data, $\mathcal{D}_{\text {obs }}$ say, can be written as the product of two components

$$
\ell\left(\mathcal{D}_{o b s} \mid \boldsymbol{\eta}\right)=\prod_{(i, j) \in \mathcal{C}} f_{i j}\left(y_{i j} \mid \eta_{i j}\right) \prod_{(i, j) \in \mathcal{P}} F_{i j}\left(P_{i j} \mid \eta_{i j}\right)
$$

taken over the sets $\mathcal{C}$ and $\mathcal{P}$, with

$$
F_{i j}\left(P_{i j} \mid \eta_{i j}\right)=\int_{y_{i j} \in P_{i j}} f_{i j}\left(y_{i j} \mid \eta_{i j}\right) d y_{i j}
$$


denoting the contribution arising from the partial information that $y_{i j}$ belongs to the set $P_{i j}$. To write down the (unconditional) likelihood for the hierarchical GLMM, we integrate over the distribution of $\boldsymbol{\eta}$ in (11):

$$
\ell\left(\mathcal{D}_{o b s} \mid \boldsymbol{\beta}, \boldsymbol{F}, \boldsymbol{\Gamma}\right)=\int_{\boldsymbol{\eta}} \ell\left(\mathcal{D}_{o b s} \mid \boldsymbol{\eta}\right) f_{0}(\boldsymbol{\eta} \mid \boldsymbol{\beta}, \boldsymbol{F}, \boldsymbol{\Gamma}) d \boldsymbol{\eta}
$$

where $f_{0}$ is the distribution of $\boldsymbol{\eta}$ given in (11). Examples of partially observed data are common in rare diseases. For example, when mapping cancer incidences, certain counties do not report the exact number of incidences if the total number is less than a known threshold. In this case the partial information is $P_{i j}=\left\{y_{i j}<\tau\right\}$ where $\tau$ is the known threshold.

\section{Default Prior Elicitation}

This section discusses the appropriate default priors on the model parameters $\boldsymbol{\beta}, \boldsymbol{H}$ and $\boldsymbol{\Gamma}$. Since each $\beta_{i}$ represents the regression effects to the mean of the observations $y_{i j}$, it is natural to elicit a standard "flat" non-informative prior on each $\beta_{i}$ for $i=1,2, \cdots, p$ :

$$
\pi_{N}(\boldsymbol{\beta}) \propto 1
$$

It is also natural to consider the Jeffrey's type non-informative prior on $\boldsymbol{\Gamma}$ of the form

$\pi_{J}(\boldsymbol{\Gamma}) \propto(\operatorname{det}(\boldsymbol{\Gamma}))^{-(p+1) / 2}$. However, the following theorem indicates that a Jeffrey's type prior leads to an improper posterior distribution.

Theorem 1. Let $\pi_{0}(\boldsymbol{H})$ be a proper prior on $\boldsymbol{H}$. The default prior specification

$$
\pi_{0}(\boldsymbol{\beta}, \boldsymbol{H}, \boldsymbol{\Gamma})=\pi_{N}(\boldsymbol{\beta}) \times \pi_{J}(\boldsymbol{\Gamma}) \times \pi_{0}(\boldsymbol{H})
$$

gives a posterior distribution on the parameters that is improper.

Proof: We refer the reader to a proof in the Appendix. The consequence of Theorem 1 is that new motivation is required for the development of a default prior on $\boldsymbol{\Gamma}$, which should make the posterior proper. We discuss the development of such a prior in the subsequent paragraphs.

Our justification for the default prior comes from looking at the conditional update of each $\eta_{i j}$ given the data $y_{i j}$ and the rest of the $\boldsymbol{\eta}$ elements (excluding $\eta_{i j}$ ). In the normal-normal case, one can explicitly derive an expression for the weights that represent the contribution of the data, $y_{i j}$, and the rest of the $\boldsymbol{\eta}$ to the conditional mean of $\eta_{i j}$. However, in the case of non-conjugate GLMMs, it is not possible to obtain a closed form expression for the weights. An approximate approach can be considered based on a quadratic expansion of 
the exponential family pdf to yield a similar analysis as in the normal-normal model. We consider the prediction of the vector $\boldsymbol{\eta}_{j}$ given $\boldsymbol{y}_{j}$ and $\boldsymbol{\eta}_{-j}$ (which are the rest of the $\eta_{i j} \mathrm{~s}$ excluding the ones at site $j$ ). The GLM approach to estimating $\boldsymbol{\eta}_{j}$ is iterative and expands the function $h_{i} \mathrm{~s}$ in (1) around the current estimate $\boldsymbol{\eta}_{j}^{*}$. From Taylor's expansion, we have

$$
h_{i}\left(\eta_{i j}\right) \approx h_{i}\left(\eta_{i j}^{*}\right)+\left(\eta_{i j}-\eta_{i j}^{*}\right) h_{i}^{(1)}\left(\eta_{i j}^{*}\right)+\frac{1}{2}\left(\eta_{i j}-\eta_{i j}^{*}\right)^{2} h_{i}^{(2)}\left(\eta_{i j}^{*}\right)
$$

Using the expansion above, the exponential family of densities can be expanded around $\boldsymbol{\eta}_{j}^{*}$ similarly as

$$
\begin{aligned}
& \prod_{i=1}^{p} \exp \left\{\eta_{i j} y_{i j}-h_{i}\left(\eta_{i j}\right)\right\} \\
\approx & \prod_{i=1}^{p} \exp \left\{\eta_{i j} y_{i j}-h_{i}\left(\eta_{i j}^{*}\right)-\left(\eta_{i j}-\eta_{i j}^{*}\right) h_{i}^{(1)}\left(\eta_{i j}^{*}\right)-\frac{1}{2}\left(\eta_{i j}-\eta_{i j}^{*}\right)^{2} h_{i}^{(2)}\left(\eta_{i j}^{*}\right)\right\} \\
\propto & \exp \left\{-\frac{1}{2}\left(\boldsymbol{\eta}_{j}-\boldsymbol{\eta}_{j}^{*}\right)^{\prime} \boldsymbol{H}_{j}^{(2)}\left(\boldsymbol{\eta}_{j}-\boldsymbol{\eta}_{j}^{*}\right)+\boldsymbol{\eta}_{j}^{\prime}\left(\boldsymbol{y}_{j}-\boldsymbol{h}_{j}^{(1)}\right)\right\}
\end{aligned}
$$

where $\boldsymbol{h}_{j}^{(1)}=\left(h_{1}^{(1)}\left(\eta_{1 j}^{*}\right), h_{2}^{(1)}\left(\eta_{2 j}^{*}\right), \cdots, h_{p}^{(1)}\left(\eta_{p j}^{*}\right)\right)^{\prime}$ is the $p \times 1$ of first derivatives, and

$$
\boldsymbol{H}_{j}^{(2)}=\operatorname{diag}\left(h_{1}^{(2)}\left(\eta_{1 j}^{*}\right), h_{2}^{(2)}\left(\eta_{2 j}^{*}\right), \cdots, h_{p}^{(2)}\left(\eta_{p j}^{*}\right)\right)
$$

is the diagonal matrix of all second derivatives of $h_{i}, i=1,2, \cdots, p$ evaluated at $\boldsymbol{\eta}_{j}^{*}$. The Taylor's expansion above allows us to revert back to the normal-normal case where by completing squares, the observational part for $\boldsymbol{\eta}_{j}$ can be derived as

$$
\exp \left\{-\frac{1}{2}\left(\boldsymbol{\eta}_{j}-\boldsymbol{\eta}_{i}^{*}\left(\boldsymbol{y}_{i}\right)\right)^{\prime} \boldsymbol{H}_{j}^{(2)}\left(\boldsymbol{\eta}_{j}-\boldsymbol{\eta}_{i}^{*}\left(\boldsymbol{y}_{i}\right)\right)\right\}
$$

with $\boldsymbol{\eta}_{i}^{*}\left(\boldsymbol{y}_{i}\right) \equiv\left(\boldsymbol{H}_{j}^{(2)}\right)^{-1}\left(\boldsymbol{y}_{j}-\boldsymbol{h}_{j}^{(1)}+H_{j}^{(2)} \boldsymbol{\eta}_{i}^{*}\right)$. The contribution from the multivariate Gaussian CAR prior in (11) (i.e., the rest of the $\boldsymbol{\eta}$ elements) is

$$
\exp \left\{-\frac{w_{j+}}{2}\left(\boldsymbol{\eta}_{j}-\boldsymbol{\eta}_{j}^{C A R}\right)^{\prime}\left(\Gamma^{-1}\right)\left(\boldsymbol{\eta}_{j}-\boldsymbol{\eta}_{j}^{C A R}\right)\right\}
$$

where $\eta_{j}^{C A R}=\boldsymbol{H} \sum_{k \in N_{j}} \frac{w_{i j}}{w_{j}} \boldsymbol{\eta}_{k}$. Combining the likelihood and prior parts, the conditional mean of $\boldsymbol{\eta}_{j}$ (again by completion of squares) turns out to be

$$
\left(\boldsymbol{H}_{j}^{(2)}+\boldsymbol{\Gamma}^{-1} w_{j+}\right)^{-1} \boldsymbol{H}_{j}^{(2)} \boldsymbol{\eta}_{j}^{*}\left(\boldsymbol{y}_{j}\right)+\left(\boldsymbol{H}_{j}^{(2)}+\boldsymbol{\Gamma}^{-1} w_{j+}\right)^{-1} \boldsymbol{\Gamma}^{-1} w_{j+} \boldsymbol{\eta}_{j}^{C A R}
$$

with (matrix) weights

$$
\boldsymbol{W}_{1 j}=\left(\boldsymbol{H}_{j}^{(2)}+\boldsymbol{\Gamma}^{-1} w_{j+}\right)^{-1} \boldsymbol{H}_{j}^{(2)} \quad \text { and } \quad \boldsymbol{W}_{2 j}=\boldsymbol{I}-\boldsymbol{W}_{1 j}
$$


corresponding to the direct estimate $\boldsymbol{y}_{j}$ and the population mean. Our proposal is to induce a prior on $\boldsymbol{\Gamma}$ such that the prior on

$$
\boldsymbol{W}_{1 j}=\left(\boldsymbol{H}_{j}^{(2)}+\boldsymbol{\Gamma}^{-1} w_{j+}\right)^{-1} \boldsymbol{H}_{j}^{(2)}=\left(\frac{\boldsymbol{H}_{j}^{(2)}}{w_{j+}}+\boldsymbol{\Gamma}^{-1}\right)^{-1} \frac{\boldsymbol{H}_{j}^{(2)}}{w_{j+}}
$$

is uniform. A similar technique was used by Daniels (1998) and Natarajan and Kass (2000) in the univariate non-spatial context. Since $\boldsymbol{W}_{1 j}$ varies with $j$, we first replace it by its average across all the sites. Thus, we set

$$
w_{0}=\frac{1}{n p} \sum_{j} \operatorname{trace}\left(\frac{H_{j}^{(2)}}{w_{j+}}\right) .
$$

Substituting $\frac{\boldsymbol{H}_{j}^{(2)}}{w_{j+}}$ by its average $w_{0} \boldsymbol{I}_{p}$ in the expression of $W_{1 j}$ above, we get the matrix $\boldsymbol{U}$ defined by

$$
\boldsymbol{U} \equiv\left(w_{0} \boldsymbol{I}_{p}+\boldsymbol{\Gamma}^{-1}\right)^{-1} w_{0} \boldsymbol{I}_{p}=\left(w_{0} \boldsymbol{\Gamma}+\boldsymbol{I}_{p}\right)^{-1} w_{0} \boldsymbol{\Gamma}
$$

Note that $0 \leq \boldsymbol{U} \leq \boldsymbol{I}_{p}$ in terms of positive definiteness with $\operatorname{det}(\boldsymbol{U})$ representing the volume of the weight matrix $\boldsymbol{U}$. We seek a prior on $\boldsymbol{\Gamma}$ that is induced by a default prior on $\operatorname{det}(\boldsymbol{U})$. The class of multivariate Beta distribution given by

$$
f(\boldsymbol{U} \mid a, b)=C(\operatorname{det}(\boldsymbol{U}))^{a-(p+1) / 2}\left(\operatorname{det}\left(\boldsymbol{I}_{p}-\boldsymbol{U}\right)\right)^{b-(p+1) / 2}
$$

with $a>(p-1) / 2$ and $b>(p-1) / 2$ forms a class of prior for $\boldsymbol{U}$. The uniform prior on the weight matrix $\boldsymbol{U}$ is obtained by setting $a=b=(p+1) / 2$. The resulting prior on $\boldsymbol{\Gamma}$ corresponding to uniform volume of $\boldsymbol{U}$ is

$$
\pi_{U V}(\boldsymbol{\Gamma})=\operatorname{det}\left(\boldsymbol{I}_{p}+w_{0} \boldsymbol{\Gamma}\right)^{-(p+1)} .
$$

This is also the prior developed in Natarajan and Kass (2000) leading to shrinkage-type estimators in the non-spatial context. The uniform volume prior is proper from Theorem 2 of Natarajan and Kass (2000).

The prior on $\boldsymbol{H}$ is induced via $\boldsymbol{F}$. The prior on $\boldsymbol{F}$ is taken to be independent of $\boldsymbol{\Gamma}$ and is elicited as follows: Writing the spectral decomposition of $\boldsymbol{F}$ as

$$
\boldsymbol{F}=Q \Lambda Q^{\prime}
$$

we put a uniform prior on $\boldsymbol{Q}$. To have positive definiteness, we put a uniform prior $U(-1,+1)$ on the eigenvalues in $\boldsymbol{\Lambda}$. The default prior on $(\boldsymbol{\beta}, \boldsymbol{F}, \boldsymbol{\Gamma})$ is thus

$$
\pi_{0}(\boldsymbol{\beta}, \boldsymbol{F}, \boldsymbol{\Gamma})=\pi_{N}(\boldsymbol{\beta}) \times \pi_{U V}(\boldsymbol{\Gamma}) \times \frac{1}{2^{p}} .
$$

For each $i=1,2, \cdots, p$, the design matrix corresponding to the $i$-th response variable is the $n \times q_{i}$ matrix $\tilde{\boldsymbol{X}}_{i}=\left(x_{i 1}, x_{i 2}, \cdots, x_{i n}\right)^{\prime}$. The submatrix $\tilde{\boldsymbol{X}}_{\mathcal{C}_{i}}$ is formed by taking all rows $j$ of $\tilde{\boldsymbol{X}}_{i}$ for which $(i, j) \in \mathcal{C}$. We state 
Theorem 2. Assume that $f_{i j}$ and $F_{i j}$ in (13) are bounded above by a constant independent of $\eta_{i j}$ for each pair $(i, j)$. Using the default prior (24), the posterior is proper if there exists $q_{i}$ linearly independent row vectors in $\tilde{\boldsymbol{X}}_{\mathcal{C}_{i}}$ for each $i=1,2, \cdots, p$ such that

$$
\int_{\boldsymbol{\Gamma}} \int_{\boldsymbol{F}} \int_{\boldsymbol{\beta}} \int_{\boldsymbol{\eta}}\left(\prod_{i=1}^{p} \prod_{j=1}^{q_{i}} f_{i j}\left(y_{i j} \mid \eta_{i j}\right)\right) f_{0}(\boldsymbol{\eta} \mid \boldsymbol{\beta}, \boldsymbol{F}, \boldsymbol{\Gamma}) d \boldsymbol{\eta} \pi_{0}(\boldsymbol{\beta}, \boldsymbol{F}, \boldsymbol{\Gamma}) d \boldsymbol{\beta} d \boldsymbol{F} d \boldsymbol{\Gamma}<\infty
$$

where $f_{0}$ and $\pi_{0}$ is, respectively, the distribution of $\boldsymbol{\eta}$ and the prior, as given in (11) and (24).

Remark 1: Under the assumptions of Theorem 2, $0 \leq f_{i j} \leq A$ and $0 \leq F_{i j} \leq B$, say. In our applications, $f_{i j}$ is taken to be either a Poisson pmf or a normal pdf. For the Poisson (or, generally for a discrete distribution), it easily follows that the bounds $A=B=1$, independent of $i$ and $j$. When $f_{i j}$ is normal with mean $\eta_{i j}$ and fixed standard deviation $\sigma_{0}$, it follows that $A=1 / \sqrt{2 \pi} \sigma_{0}$ and $B=1$, thus independent of $i$ and $j$ again. Generally for densities, the bound $A$ needs to be established on a case-by-case basis.

Remark 2: Theorem 2 shows that propriety can be achieved if there are at least $q_{i}$ sites on the lattice for which the $y_{i j}$ s are completely observed. The only further check that we have to perform is to see if the design matrix corresponding to those sites $\tilde{\boldsymbol{X}}_{\mathcal{C}_{i}}$ is non-singular. This is a requirement for each $i$, so the conditions of Theorem 2 can be checked separately for each $i=1,2, \cdots, p$.

\section{Bayesian Inference}

The Gibbs sampler is a natural method for posterior simulations in the case of GLMMs, and is also the method utilized for our spatial models. A slightly different (yet equivalent) parametrization of the spatial multivariate GLMM is considered subsequently. Instead of $\boldsymbol{\Gamma}^{-1 / 2}$, the lower triangular matrix $\boldsymbol{L}$ obtained from the Cholesky decomposition of $\boldsymbol{\Gamma}^{-1}$ is used. Also, we define the matrix $\boldsymbol{B} \equiv \boldsymbol{L} \boldsymbol{Q}$ with entries $\boldsymbol{B}=\left(\left(b_{u v}\right)\right)_{u, v=1}^{p}$ where $\boldsymbol{Q}$ is the orthogonal matrix from the spectral value decomposition of $\boldsymbol{F}$. The following string of equalities demonstrate that $\boldsymbol{D}$ is a function of $\boldsymbol{B}$ and $\boldsymbol{\Lambda}$ : We have

$$
\begin{aligned}
\boldsymbol{D}^{-1} & =\left(\boldsymbol{I}_{n} \otimes \boldsymbol{L}\right)\left(\boldsymbol{M} \otimes \boldsymbol{I}_{p}-\boldsymbol{W} \otimes \boldsymbol{F}\right)\left(\boldsymbol{I}_{n} \otimes \boldsymbol{L}^{\prime}\right) \\
& =\left(\boldsymbol{I}_{n} \otimes \boldsymbol{L}\right)\left(\boldsymbol{M} \otimes \boldsymbol{I}_{p}-\boldsymbol{W} \otimes \boldsymbol{Q} \boldsymbol{\Lambda} \boldsymbol{Q}^{\prime}\right)\left(\boldsymbol{I}_{n} \otimes \boldsymbol{L}^{\prime}\right) \\
& =\left(\boldsymbol{I}_{n} \otimes \boldsymbol{L} \boldsymbol{Q}\right)\left(\boldsymbol{M} \otimes \boldsymbol{I}_{p}-\boldsymbol{W} \otimes \boldsymbol{\Lambda}\right)\left(\boldsymbol{I}_{n} \otimes \boldsymbol{Q}^{\prime} \boldsymbol{L}^{\prime}\right) \\
& =\left(\boldsymbol{I}_{n} \otimes \boldsymbol{B}\right)\left(\boldsymbol{M} \otimes \boldsymbol{I}_{p}-\boldsymbol{W} \otimes \boldsymbol{\Lambda}\right)\left(\boldsymbol{I}_{n} \otimes \boldsymbol{B}^{\prime}\right) .
\end{aligned}
$$

The enormous advantage of the re-parametrization in terms of $\boldsymbol{B}$ is that the entries of $\boldsymbol{B}$ are unconstrained. Note that it is possible to obtain $\boldsymbol{Q}$ and $\boldsymbol{L}$ uniquely from $\boldsymbol{B}$ using the 
QR decomposition of $\boldsymbol{B}^{\prime}=\mathcal{Q R}$ where $\mathcal{Q}$ is orthogonal and $\mathcal{R}$ is upper triangular. It follows that $\boldsymbol{L}=\mathcal{R}^{\prime}$ and $\boldsymbol{Q}=\mathcal{Q}^{\prime}$.

The four main steps of the Gibbs sampler are:

- Update $\eta_{i j}$ : The update of $\eta_{i j}$ is carried out based on the following (conditional) posterior density of $\eta_{i j}$ given the rest of the parameters:

$$
\pi\left(\eta_{i j} \mid \cdots\right) \propto \exp \left\{\eta_{i j} y_{i j}-h_{i}\left(\eta_{i j}\right)-\frac{A_{i j}}{2}\left(\eta_{i j}-\eta_{i j}^{*}\right)^{2}\right\}
$$

where $\eta_{i j}^{*} \equiv x_{i j}^{\prime} \beta_{i}+\epsilon_{i j}^{*}$, and $\epsilon_{i j}^{*}$ has the expression $\epsilon_{i j}^{*}=\frac{1}{\sum_{v=1}^{p} b_{i v}^{2}} \epsilon_{0}$,

$$
\epsilon_{0}=\underbrace{\sum_{v=1}^{p} b_{i v}^{2} \lambda_{v} \sum_{k \in N_{j}} \frac{w_{j k}}{w_{j+}} \epsilon_{i k}}_{(1)}-\underbrace{\sum_{v=1}^{p} \sum_{u=1, u \neq i}^{p} b_{i v} b_{u v} \epsilon_{u j}}_{(2)}+\underbrace{\sum_{v=1}^{p} \sum_{u=1, u \neq i}^{p} b_{i v} b_{u v} \lambda_{v} \sum_{k \in N_{j}} \frac{w_{j k}}{w_{j+}} \epsilon_{u k}}_{(3)}
$$

with $\epsilon_{u v}=\eta_{u v}-x_{u v}^{\prime} \beta_{u v}$ for all $(u, v)=1,2, \cdots, p$ except for $(u, v)=(i, j)$, and $A_{i j}=$ $w_{j+} \sum_{v=1}^{p} b_{i v}^{2}$. The updating formula in (27) is based on three components: The first component (terms in (1) above) involve $\eta_{i k}$ values for spatial sites $k \in N_{j}$ for the same variable index $i$, the second component involves $\eta_{u j}$ for the other variables $u \neq i$ but for the same spatial location $j$, whereas the third component involves $\eta_{u k}$ for variables other than $i$ and sites in $k \in N_{j}$. The update of $\eta_{i j}$ is based on histogramming the conditional posterior density in (26) for each fixed pair $(i, j)$ and cycling through all the combinations of $(i, j) \in\{(1,1),(1,2), \cdots,(1, n), \cdots,(p, n)\}$. This is the $\boldsymbol{\eta}_{i j}$ updating step when $y_{i j}$ is completely observed, that is, for $(i, j) \in \mathcal{C}$. When $y_{i j}$ is missing, the first term in equation (26) will be absent. The standard method of analyzing partially observed $y_{i j}$ is to treat it as missing and update the value based on the truncated distribution $f_{i j}\left(\cdot \mid \eta_{i j}\right)$ given that $y_{i j} \in P_{i j}$.

- Update $\boldsymbol{\beta}$ : The update of $\boldsymbol{\beta}$ requires a re-ordering of the variables involved. For each $i=1,2, \cdots, p$, define $\boldsymbol{\eta}_{i}^{r}$ and $\boldsymbol{\epsilon}_{i}^{r}$ to be the $n \times 1$ vectors corresponding to the $i$-th variable, that is, $\boldsymbol{\eta}_{i}^{r}=\left(\eta_{i 1}, \eta_{i 2}, \cdots \eta_{i n}\right)^{\prime}$ and $\boldsymbol{\epsilon}_{i}^{r}=\left(\epsilon_{i 1}, \epsilon_{i 2}, \cdots \epsilon_{i n}\right)^{\prime}$. Also, let $\boldsymbol{\eta}^{r} \equiv$ $\left(\left(\boldsymbol{\eta}_{1}^{r}\right)^{\prime},\left(\boldsymbol{\eta}_{2}^{r}\right)^{\prime}, \cdots,\left(\boldsymbol{\eta}_{p}^{r}\right)^{\prime}\right)$ and $\boldsymbol{\epsilon}^{r} \equiv\left(\left(\boldsymbol{\epsilon}_{1}^{r}\right)^{\prime},\left(\boldsymbol{\epsilon}_{2}^{r}\right)^{\prime}, \cdots,\left(\boldsymbol{\epsilon}_{p}^{r}\right)^{\prime}\right)$ denote the $n p \times 1$ vector of reordered entries from $\boldsymbol{\eta}$ and $\boldsymbol{\epsilon}$, respectively. The covariance matrix of $\boldsymbol{\epsilon}^{r}$ is subsequently a re-ordered version of $\boldsymbol{D}$ given by

$$
\left(\boldsymbol{D}^{r}\right)^{-1}=\left(\boldsymbol{B} \otimes \boldsymbol{I}_{n}\right)\left(\boldsymbol{I}_{p} \otimes \boldsymbol{M}-\boldsymbol{\Lambda} \otimes \boldsymbol{W}\right)\left(\boldsymbol{B}^{\prime} \otimes \boldsymbol{I}_{n}\right)
$$

Also, let $\tilde{\boldsymbol{X}}=\left(\left(\right.\right.$ Block Diagonal $\left.\left.\left\{\tilde{\boldsymbol{X}}_{i}\right\}\right)\right)$ denote the block diagonal matrix consisting of the design matrices for the $i$-th response variable for $i=1,2, \cdots, p$. The conditional 
posterior distribution of $\boldsymbol{\beta}$ is multivariate normal with mean $\boldsymbol{\mu}_{\boldsymbol{\beta}}$, and covariance matrix $\boldsymbol{S}_{\boldsymbol{\beta}}$ where

$$
\boldsymbol{\mu}_{\boldsymbol{\beta}}=\left(\tilde{\boldsymbol{X}}^{\prime}\left(\boldsymbol{D}^{r}\right)^{-1} \tilde{\boldsymbol{X}}\right)^{-1}\left(\tilde{\boldsymbol{X}}^{\prime}\left(\boldsymbol{D}^{r}\right)^{-1} \boldsymbol{\eta}^{r}\right) \quad \text { and } \quad \boldsymbol{S}_{\boldsymbol{\beta}}=\left(\tilde{\boldsymbol{X}}^{\prime}\left(\boldsymbol{D}^{r}\right)^{-1} \tilde{\boldsymbol{X}}\right)^{-1}
$$

respectively.

- Update $\boldsymbol{\Lambda}$ : An enormous advantage of the re-parametrization in terms of $\boldsymbol{B}$ and $\boldsymbol{\Lambda}$ earlier is when updating $\Lambda$ : The diagonal entries of $\boldsymbol{\Lambda}$ can be updated independently of each other. Consider the $p \times n$ matrix, $\Upsilon$, constructed by putting $\epsilon_{i j}$ in its $i$-th row and $j$-th column entry. Define a new matrix $p \times n$ matrix $\boldsymbol{E}$ as

$$
\boldsymbol{E}=\boldsymbol{B}^{\prime} \Upsilon
$$

and let $e_{i}^{\prime}$ be the $i$-th row of $\boldsymbol{E}$, for $i=1,2, \cdots, p$. The conditional posterior density of $\lambda_{k}$ is given by

$$
\pi(\lambda \mid \cdots) \propto \exp \left\{-\frac{1}{2} e_{k}^{\prime}(\boldsymbol{M}-\lambda \boldsymbol{W}) e_{k}\right\}(\operatorname{det}(\boldsymbol{M}-\lambda \boldsymbol{W}))^{1 / 2}
$$

in the range of $-1 \leq \lambda \leq 1$ independently for each $k=1,2, \cdots, p$. The update of $\lambda_{k}$ is based on histogramming the conditional posterior density in (31) for each fixed $k$ and cycling through all $k=1,2, \cdots, p$.

- Update $\boldsymbol{B}$ : The conditional posterior density of $\boldsymbol{B}$ has the expression

$$
\begin{aligned}
\pi(\boldsymbol{B} \mid \cdots) \propto & \exp \left\{-\frac{1}{2} \sum_{k=1}^{p} e_{k}^{\prime}\left(\boldsymbol{M}-\lambda_{k} \boldsymbol{W}\right) e_{k}\right\} \times \operatorname{det}\left(w_{0} \boldsymbol{I}+\boldsymbol{B} \boldsymbol{B}^{\prime}\right)^{-(p+1)} \\
& \times \operatorname{det}\left(\boldsymbol{B} \boldsymbol{B}^{\prime}\right)^{(n+1) / 2}
\end{aligned}
$$

where $e_{k}^{\prime} \equiv e_{k}^{\prime}(\boldsymbol{B})$ is as defined in (30) but now viewed as a function of $\boldsymbol{B}$. The latter part of the conditional density in (32) is the contribution of the default prior on $\boldsymbol{\Gamma}$. The update of $\boldsymbol{B}$ is carried out by updating each entry $b_{u v}$ one at a time. The conditional posterior density of $b_{u v}$ is the same (upto a constant of proportionality) as in (32). The actual update of $b_{u v}$ is performed by histogramming the density in (32) and cycling through all combinations $(u, v)$ with $u, v=1,2, \cdots, p$.

\section{Experimental Results}

\subsection{Simulation study}

We have conducted extensive simulation studies to check the performance of our methodology. The experimental settings closely mimic county-level data for southern lower Michigan 
obtained from the SEER database. We took $p=2$ with $y_{1 j}$ and $y_{2 j}$ representing binomial responses on $n=40$ spatial sites with neighborhood structure determined by the adjacency information among 40 southern lower Michigan counties. Given $\boldsymbol{\eta}$, the observed data $y_{i j} \sim \operatorname{Binomial}\left(T_{i j}, \frac{e^{\eta_{i j}}}{1+e^{\eta_{i j}}}\right)$, for $i=1,2$ and $j=1,2, \cdots, 40$, independent of each other; $T_{i j}$ is the total number of trials selected independently from a Uniform $(22,48)$ distribution. The distribution of $\boldsymbol{\eta}$ is multivariate Gaussian CAR with the following true parameter specifications: $\boldsymbol{\beta}_{1}=(-1,0.3)^{\prime}, \boldsymbol{\beta}_{2}=(-0.5,-0.2)^{\prime}$,

$$
\boldsymbol{\Gamma}=\left(\begin{array}{cc}
0.5 & 0.1 \\
0.1 & 0.125
\end{array}\right) \quad \text { and } \quad \boldsymbol{F}=\left(\begin{array}{cc}
0.7 & -0.1 \\
-0.1 & 0.2
\end{array}\right) \text {. }
$$

The first components of $\boldsymbol{\beta}_{1}$ and $\boldsymbol{\beta}_{2}$ correspond to the intercept terms. Here $q_{1}=q_{2}=2$ and additional covariate information is gathered from independent normal distributions: $X_{1} \sim N\left(0, \sigma^{2}=0.3\right)$ and $X_{2} \sim N\left(1, \sigma^{2}=0.5\right)$.

Two types of priors were considered for $\boldsymbol{\Gamma}$, namely, (a) the default prior $\pi_{U V}(\boldsymbol{\Gamma}) \propto$ $\operatorname{det}\left(\boldsymbol{I}_{\boldsymbol{p}}+\boldsymbol{\omega}_{\mathbf{0}} \boldsymbol{\Gamma}\right)^{-(p+1)}$ and (b) the proper inverse Wishart given by $\pi_{I W}(\boldsymbol{\Gamma})=I W(\rho, \rho \boldsymbol{A})$, where $\rho \geq p$. The inverse Wishart distribution is a generalization of the inverse gamma for the variance parameter in a multivariate setting. If $\boldsymbol{\Gamma} \sim I W(m, \Psi)$, the expectation and variance of entries of $\boldsymbol{\Gamma}$ are given by $E\left(\boldsymbol{\Gamma}_{k l}\right)=\Psi_{k l} /(m-p-1)$ and $\operatorname{var}\left(\boldsymbol{\Gamma}_{k l}\right)=$ $\frac{(m-p+1) \boldsymbol{\Psi}_{k l}^{2}+(m-p-1) \boldsymbol{\Psi}_{k k} \boldsymbol{\Psi}_{l l}}{(m-p)(m-p-1)^{2}(m-p-3)}$ where $\boldsymbol{\Gamma}_{k l}$ and $\boldsymbol{\Psi}_{k l}$ are the $(k, l)$-th entries of $p \times p$ matrices $\boldsymbol{\Gamma}$ and $\boldsymbol{\Psi}$, respectively. When $\rho$ is large, $E(\boldsymbol{\Gamma}) \approx \boldsymbol{A}$, and $\operatorname{var}\left(\boldsymbol{\Gamma}_{i j}\right) \approx 1 / \rho$, leading to a high concentration of probability around the initial guess of $\boldsymbol{A}$. Thus, this prior does not represent non-informative prior knowledge. Prior (b) was proposed by Sain and Cressie (2007) as the choice of vague prior for $\Gamma$ when $\rho$ is large, which is not the case (actually, Sain and Cressie (2007) put a prior on $\boldsymbol{\Gamma}^{-1}$ which is $\operatorname{Wishart}\left(\rho,(\rho \boldsymbol{A})^{-1}\right)$, but this is equivalent to choice (b) since $\boldsymbol{\Gamma} \sim I W(m, \Psi) \quad$ iff $\left.\quad \boldsymbol{\Gamma}^{-1} \sim W\left(m, \Psi^{-1}\right)\right)$.

The priors (a) and (b) for $\boldsymbol{\Gamma}$ above in turn induce priors on $\boldsymbol{B}$. This is based on the transformations $\boldsymbol{\Gamma} \rightarrow \boldsymbol{\Gamma}^{-\mathbf{1}}, \boldsymbol{\Gamma}^{-\mathbf{1}} \rightarrow \boldsymbol{L} \boldsymbol{L}^{\prime}$, and $(\boldsymbol{L}, \boldsymbol{Q}) \rightarrow \boldsymbol{B}$. The derivation of the Jacobian for the composition transformation from $(\boldsymbol{\Gamma}, \boldsymbol{Q}) \rightarrow \boldsymbol{B}$ is given in the Appendix. The priors on $\boldsymbol{B}$ turn out to be (i) $\pi_{U V}(\boldsymbol{B})=\operatorname{det}\left(\boldsymbol{\omega}_{\mathbf{0}} \boldsymbol{I}_{\boldsymbol{p}}+\boldsymbol{B} \boldsymbol{B}^{\prime}\right)^{-(p+1)} \operatorname{det}\left(\boldsymbol{B} \boldsymbol{B}^{\prime}\right)^{1 / 2}$, (ii) $\pi_{I W}(\boldsymbol{B})=$ $\exp \left\{-\frac{\rho}{2} \operatorname{tr}\left(\boldsymbol{A} \boldsymbol{B} \boldsymbol{B}^{\prime}\right)\right\} \times \operatorname{det}\left(\boldsymbol{B} \boldsymbol{B}^{\prime}\right)^{\frac{\rho-p}{2}}$ respectively, for the priors (a) and (b) for $\boldsymbol{\Gamma}$. Prior choices for $\boldsymbol{\beta}$ are (i) the default non-informative constant prior $\pi_{N}(\boldsymbol{\beta}) \propto 1$, and (ii) the proper subjective prior $\pi_{G}\left(\boldsymbol{\beta}_{k}\right) \sim N\left(0, \sigma_{k}^{2} \boldsymbol{I}_{q_{k}}\right)$ independently for each $k=1,2, \cdots, p$. Using (ii), it is easy to see that the posterior for $\boldsymbol{\beta}$ is $N(\boldsymbol{\mu}, \boldsymbol{S})$ where

$$
\boldsymbol{\mu}=\boldsymbol{S} \boldsymbol{X}^{\prime}\left(\boldsymbol{D}^{r}\right)^{-1} \boldsymbol{\eta}^{r}, \boldsymbol{S}^{-1}=\boldsymbol{X}^{\prime}\left(\boldsymbol{D}^{\boldsymbol{r}}\right)^{-1} X+\Sigma^{-1} .
$$

Here, $\boldsymbol{\Sigma}=$ Block Diagonal $\left(\sigma_{k}^{2} \boldsymbol{I}_{q_{k}}\right)$. Thus, we investigate the following three prior choices: (I) $\pi_{U V}(\boldsymbol{B})$ and $\pi_{N}(\boldsymbol{\beta})$ and $(\mathrm{II}) \pi_{I W}(\boldsymbol{B})$ and $\pi_{G}\left(\boldsymbol{\beta}_{k}\right)$ with $\rho=5, A=I_{p}$ and $\sigma_{k}^{2}=100$, and (III) 
$\pi_{I W}(\boldsymbol{B})$ and $\pi_{G}\left(\boldsymbol{\beta}_{k}\right)$ with $\rho=100,000, A=I_{p}$ and $\sigma_{k}^{2}=100$. That is, (I) is our proposed default prior, (II) is a proper prior with small $\rho$ and (III) is a prior with large $\rho$. Note that (II) is weakly informative whereas (III) is highly informative.

The Gibbs sampler was run for 10,000 iterations and checked for convergence using traceplots and the $R$-statistic of Gelman and Rubin (1992). We established convergence for all the experiments by 5,000 iterations; all diagnostic plots are satisfactory but are suppressed to save space. Outputs from the Gibbs chains were used to compute different statistics to validate and compare the proposed Bayesian methodology. The three prior choices are compared in terms of their ability to derive consistent estimation and prediction results. The deviation measures of comparisons are (1) relative mean absolute deviation (RMAD), (2) mean square error (MSE), (3) empirical 90\% HPD coverage probabilities (CP), and (4) width of the $90 \% \mathrm{HPD}$ set $(\mathrm{W})$. Formulas for a generic quantity $\theta$ are given by $R M A D_{\theta}=\left(E(\theta)-\theta_{0}\right) / \theta_{0}, M S E_{\theta}=E\left(\theta-\theta_{0}\right)^{2}, C P_{\theta}=P\left\{\theta_{0} \in H P D(\theta)\right\}$ and $W$ is the width of $H P D(\theta)$; in the RHS of each expression, $\theta$ represents a sample (or samples) from the posterior, $\operatorname{HPD}(\theta)$ is the $90 \%$ HPD set calculated based on $\theta$ samples and $\theta_{0}$ is the true value set in the simulation experiments. We used 500 replications in each experiment and report the averages of the deviation measures above. The computational time for all the 500 replications in each experimental setup is approximately $20-25$ hours. All computations were carried out using an HP ProLiant DL160 machine (a cluster of nodes) with 8 Intel Xeon cores and 24GB of memory at the High Performance Computing Center (HPCC) in Michigan State University.

Table 1 reports a summary of all deviation measures. For convenience of understanding the results, we report averages over specific components of the unknown parameters; for example, the $\boldsymbol{\beta}$ column reports the average over all $\beta$ components, $\beta_{11}, \beta_{12}, \beta_{21}, \beta_{22}$, and similarly for the other parameters $\boldsymbol{\Gamma}$ and $\boldsymbol{F}$. The last column reports averages over all $40 \times 2=80$ predicted values for $\boldsymbol{\eta}$; the deviation measures are calculated based on $\boldsymbol{\eta}_{0}$, the true values generated in each replication. Entries in Table 1 clearly shows the sensitivity of standard prior distributions (i.e., priors (II) and (III)) used in the literature. For example, for a wrong choice of $\rho$ and $\boldsymbol{A}$ in $\pi_{I W}$, the coverage can be even 0 along with high MSE and RMAD. This effect can be reduced with a more sensible prior choice, for example, choice (II). On the other hand, $\pi_{U V}$ always provides sensible results. One might notice that the sensitivity of priors (II) and (III) is highest for $\boldsymbol{\Gamma}$ compared to the other columns in Table 1. This is due to the fact that the prior for $\boldsymbol{\Gamma}$ changes significantly for the three choices (I-III) whereas we always use the default uniform prior for $\boldsymbol{F}$. The regression parameters $\boldsymbol{\beta}$ are less affected by the prior choice compared to $\boldsymbol{\Gamma}$ due to the fact that $\boldsymbol{\beta}$ is related to the mean parameter with large prior variance while $\boldsymbol{\Gamma}$ is related to dispersion. Nevertheless, the standard choice of Gaussian prior on $\boldsymbol{\beta}$ also appears to be somewhat sensitive, but not to 
the extent of $\boldsymbol{\Gamma}$. Although the $\boldsymbol{\eta}$ components are not fixed model parameters (i.e., they vary from county to county), their inference can also be sensitive to the different prior choices. To explain the discrepancies in the $\boldsymbol{\Gamma}$ entries, Figure 1 plots the posterior densities of $\Gamma_{22}$, the $(2,2)$-th entry of $\boldsymbol{\Gamma}$, corresponding to the three different prior choices for a arbitrarily chosen replicate. Note that under prior (III), the prior mean is $I_{2}$ whereas the prior variance is $10^{-5}$ making it highly concentrated on a value different from the true $\Gamma_{22}$; while we understand that the small prior variance is unreasonable, this choice is not uncommon (see, for example, Sain and Cressie, 2007). The situation improves under prior (II) where the prior mean is the same but the prior variance is 0.2 which gives a comparatively higher prior mass around the true $\Gamma_{22}$. Overall, the proposed default prior $\pi_{U V}$ performed well in all respects. This prior is thus a robust choice. We have also explored with another choice of $\boldsymbol{\Gamma}$, namely

$$
\boldsymbol{\Gamma}=\left(\begin{array}{cc}
10 & 6 \\
6 & 5
\end{array}\right)
$$

The results are similar to one discussed here and is therefore not presented. The componentwise univariate spatial analysis was carried out and, as expected, the multivariate analysis had superior performance.

We also performed similar experiments with $10 \%$ missing observations. The results are reported in Table 2. Comparative trends similar to the complete data case with priors (I-III) are also observed here.

\subsection{Real data examples}

The Bayesian inferential framework developed in this paper is applied to study bivariate dependence of a number of health-socio-economic indicators in the state of Michigan. Two studies are conducted with different pairs of response variables: (1) lung cancer mortality incidence and poverty, and (2) lung cancer mortality and air quality index (AQI) measurements. Study (1) and (2) illustrate the complete and missing data applications, respectively.

We consider lung cancer mortality counts over a period 2001-2005 in Michigan counties obtained from SEER program (SEER*Stat Database: Mortality - All COD, Aggregated With County,Total U.S. (1990-2005) < Katrina/Rita Population Adjustment>). A poverty and other socio-economic county attributes are also obtained from the SEER program which is based on US Census 2000 (SEER*Stat Database: County Attributes - Total U.S., 19692005 Counties, www.seer.cancer.gov/seerstat/variables/countyattribs ). The AQI measurements are obtained from the US Environmetal Protection Agency (EPA) AQI report site (http://www.epa.gov/air/data/monaqi.html?st ${ }^{\sim}$ MI Michigan). We consider an average of median AQIs over a period 2001-2005. The AQI is a daily index value calculated for each air pollutant measured. The highest of those index values is the AQI 
value, and the pollutant responsible for the highest index value is the "Main Pollutant". The criteria pollutants used to calculate AQI are: CO (Carbon monoxide), $\mathrm{NO}_{2}$ (Nitrogen dioxide), $\mathrm{O}_{3}$ (Ozone), $\mathrm{SO}_{2}$ (Sulfur dioxide), $\mathrm{PM}_{2.5}$ (Particulate matter smaller than 2.5 micrometers) and $\mathrm{PM}_{10}$ (Particulate matter smaller than 10 micrometers). The highest possible AQI value is 500. A day having an AQI value 0 through 50 is considered 'Good' and a day having an AQI value 0 through 50 is considered 'Moderate'. Total 32 counties in Michigan have AQI measurements over a period 2001-2005 and the range of the AQI values in Michigan counties fall in either 'Good' or 'Moderate' category. Emission variables, in particular, $\mathrm{PM}_{2.5}$ is obtained from US EPA's National Emission Inventory (NEI) database

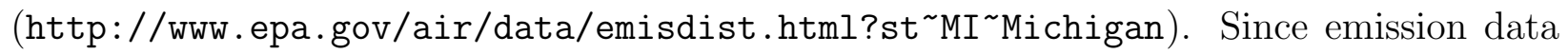
in this website is available only up to 2002, we consider the average of 2001-2002 emission data. The unit is tons per county. In each application, the Gibbs sampler was run for 10,000 iterations and checked for convergence as in the simulated data. Posterior samples are obtained from the Gibbs chains for computing the mean, standard deviation and $90 \%$ highest posterior density (HPD) intervals for all the parameters.

\subsubsection{Complete Data Example: Study 1}

The mortality of lung cancer, the first component of the response variable in Study 1, is rare enough relative to the population in the 68 counties of lower Michigan that a Poisson distribution is appropriate. We write the model (conditional on $\eta_{1 j}$ ) as

$$
y_{1 j} \stackrel{\text { ind }}{\sim} \operatorname{Poisson}\left(E_{j} e^{\eta_{1 j}}\right), i=1,2 \text { and } j=1,2, \cdots, 68
$$

where $y_{1 j}$ measures the observed number of deaths in county $j$ and $E_{j}$ is the estimated population at risk; we assume $E_{j}$ is known and give a way for calculating them later. The poverty count, $y_{2 j}$, is taken to be the second component of the response variable in Study 1. We model $y_{2 j}$ as a Binomial with the number of trials being the total county population and success probability $\frac{e^{\eta_{2 j}}}{1+e^{\eta_{2 j}}}$. The associated covariates for $y_{1 j}$ are the intercept, $\mathrm{PM}_{2.5}$, the extents of urbanization, non-white population and non-industry (these are measured as proportions). Covariates for poverty are the intercept, the extents of urbanization and non-industry. Thus, $q_{1}=5$ and $q_{2}=3$.

To calculate $E_{j}$, we take each county's age distribution into account, which is available from U.S. Census 2000. The expected age-adjusted number of deaths due in county $j$ is

$$
E_{j}=\sum_{k=1}^{m} \omega^{k} N_{j}^{k},
$$

for $j=1,2, \cdots, 68$ where $\omega^{k}=\sum_{j=1}^{68} D_{j}^{k} / \sum_{j=1}^{68} N_{j}^{k}$ is the age-specific death rate due to lung cancer for age group $k$ and $N_{j}^{k}$ is the total population at risk in county $j$ for age group $k$. 
The county level maps of the age-adjusted standardized mortality ratios (SMRs), $Y_{1 j} / E_{j}$ for lung cancer shown in Figure 2 exhibit evidence of correlation over space. Figure 2 also gives the spatial distribution of poverty levels which can be seen to be highly spatially correlated with lung cancer (simple correlation between lung cancer and poverty is around 0.4).

We present summary conclusions of our analysis. The standard errors of the parameters estimates for prior (I) are smaller compared to priors (II) and (III). For example, the average standard error of regression coefficients for lung cancer is 0.61 under prior (I), and 0.65 and 1.28 for (II) and (III), respectively. For the variance component parameters, the standard errors and widths of HPD sets are comparable under priors (I) and (II). Note that the inference for $\boldsymbol{\Gamma}$ is highly misleading for prior (III) since most of the posterior probability is concentrated around the prior mean. Another difference is that the covariate $\mathrm{PM}_{2.55}$ (related to the particle matter in the air) for lung cancer incidence is positive and significant under (I), whereas it is insignificant under priors (II) and (III). For prior (III), the posterior mean takes a negative value which is not very realistic. For brevity, other statistics along with the smooth map of $\boldsymbol{\eta}$ are suppressed.

\subsubsection{Missing Data Example: Study 2}

In Study 2, AQI is taken as the second component of the response variable in place of poverty. Air pollutant monitoring stations are sparsely located in 32 out of 68 lower Michigan counties, and thus, constitutes missing information. The covariates for AQI are the intercept and the non-industrialization status of the county $\left(q_{2}=2\right)$. We take $y_{2 j}$ to be normally distributed with mean $\eta_{2 j}$ and fixed standard deviation $\sigma_{0}$, estimated using our data and set at 0.1 .

Results for the standard errors and width of HPD sets for the parameters are similar to the complete data case. There are two striking features in this application. First, the extent of urbanization for lung cancer incidence is negative and significant under (I) whereas it is positive under the other two priors (which may not be reasonable). Second, the regression coefficient for racial segregation (non-white) is significant under (I) and (II) but not under (III). This shows the sensitivity of the subjective elicitation under the missing data setup as well.

\section{Conclusion}

The Bayesian inferential framework is perhaps the only solution available for analyzing hierarchical spatial multivariate data. In the absence of reliable subjective information, the use of Jeffreys type non-informative priors or diffuse conjugate priors is popular. However, in the context of the hierarchical spatial multivariate CAR models, we have shown that 
none of these priors will work; the Jeffreys prior yields a posterior that is improper whereas the diffuse conjugate prior is highly sensitive. This characteristic has also been observed in Natarajan and Kass (2000) in a simpler setup, namely in univariate GLMMs without any

spatial components. This led us to elicit priors on the model parameters that will be close to Jeffreys but still yield a proper posterior for inference.

The development of prior elicitation can be thought of as an extension of Natarajan and Kass (2000) in the spatial context. Besides the prior development, we propose some innovative computational techniques for the Gibbs implementation. Suitable transformations are made on the parameters which avoid sampling from restricted domains, thus providing more stability and efficiency in the Gibbs steps. The methodology has been extended to the case of missing responses in the multi-dimensional setup.

We have carried out extensive simulation studies to establish the superiority of the proposed methodology. As we have mentioned in the Introduction, the motivation of this work came from Michigan SEER data analysis. We have provided two real examples briefly merely for illustration. Both examples support the use of the newly proposed prior rather than commonly used Jeffreys or diffuse conjugate priors.

\section{References}

Carlin, B.P. and Banerjee, S. (2003), Hierarchical multivariate CAR models for spatiotemporally correlated data., In: Bernado, J.M. et al. (Eds) Bayesian Statistics 7, Oxford University Press, Oxford, 45-63.

Gelfand, A. and Vounatsou, P. (2003), Proper multivariate conditional autoregressive models for spatial data analysis, Biostatistics, 4, 11-25.

Gelman, A., and Rubin, D. (1990). Inference from iterative simulation using multiple sequences (with discussion). Statist. Sci., 7, 457-511.

Greco, F. P., and Trivisano, C. (2009). A multivariate CAR model for improving the estimation of relative risks. Statist. Med., 28, 1707-1724.

Jin, X., Carlin, B.P., and Banerjee, S. (2005), Generalized hierarchical multivariate CAR models for areal data, Biometrics, 61, 950-961.

Jin, X., Banerjee, S. and Carlin, B.P. (2007), Order-free coregionalized areal data models with applications to multiple disease mapping, J.R. Statist. Soc : Ser. B (Statistical Methodology), 69, 817 - 838. 
Kim, H.D. Sun, D. and Tsutakawa, R. (2001), A bivariate Bayes method for improving the estimates of mortality rates with a twofold conditional autoregressive model, J. Amer. Statist. Assoc., 96, 1506-1521.

Mardia, K.S. (1988), Multi-dimensional multivariate Gaussian Markov random fields with application to image processing, J. Mult. Anal., 24, 265-284.

McCullagh, P., and Nelder, J.A. (1989). Generalized Linear Models (2nd ed.), London: Chapman and Hall.

Muirhead (1982). Aspects of Multivariate Statistical Theory, John Wiley \& Sons Canada.

Natarajan, R. and Kass, R (2000), Reference Bayesian methods for generalized linear mixed models, J. Mult. Anal., 95, 227-237.

Sain, S. (2009), Parameter estimation for multivariate spatial lattice models, To appear in Biometrics.

Sain, S. and Cressie, N. (2007), A spatial model for multivariate lattice data, J. Economet., 140, 226-259.

Zhang, Y., Hodges, J. S. and Banerjee, S. (2009), Smoothed ANOVA with spatial effects as a competitor to MCAR in multivariate spatial smoothing, Ann. Appl. Statist., 3, 1805-1830.

\section{Appendix}

Proof of the positive definiteness of $\boldsymbol{D}$ : A necessary and sufficient condition for $\boldsymbol{D}$ to be positive definite (pd) is the expression for $\boldsymbol{D}^{-1}$ to be positive definite. Since $\boldsymbol{\Gamma}^{-1 / 2}$ is pd and hence non-singular, it follows from (10) that $\left(\boldsymbol{M} \otimes \boldsymbol{I}_{p}-\boldsymbol{W} \otimes \boldsymbol{F}\right)$ should be positive definite. The eigenvalues of $\left(\boldsymbol{M} \otimes \boldsymbol{I}_{p}-\boldsymbol{W} \otimes \boldsymbol{F}\right)$ is the same as the eigenvalues of $\left(\boldsymbol{M} \otimes \boldsymbol{I}_{p}-\boldsymbol{W} \otimes \boldsymbol{\Lambda}\right)$, which is the collection of all eigenvalues of $\boldsymbol{M}-\lambda_{k} \boldsymbol{W}$, for $k=1,2, \cdots, p$. Now, requiring that $\boldsymbol{M}-\lambda_{k} \boldsymbol{W}$ be diagonally dominant (which implies positive definiteness), it follows that

$$
\left|\lambda_{k}\right| \sum_{l \in N_{j}} w_{j l}<w_{j+} \quad \Rightarrow \quad\left|\lambda_{k}\right| w_{j+}<w_{j+} \quad \Rightarrow \quad\left|\lambda_{k}\right|<1
$$

for all $k=1,2, \cdots, p$.

Proof of Theorem 1: In order to show that the posterior is improper, it is enough to show that the marginal of $\mathbf{y} \equiv\left(\mathbf{y}_{1}^{\prime}, \mathbf{y}_{2}^{\prime}, \cdots, \mathbf{y}_{n}^{\prime}\right)^{\prime}$ does not exist; that is, the integration with 
respect to the variable $\boldsymbol{\eta}$ and the parameters $\boldsymbol{H}, \boldsymbol{\Gamma}$ and $\boldsymbol{\beta}$ is infinity. The marginal of $\mathbf{y}$ has the expression

$$
\begin{aligned}
\mathbf{m}(\mathbf{y})= & \int_{\boldsymbol{\eta}} \int_{\boldsymbol{H}} \int_{\boldsymbol{\Gamma}} \int_{\boldsymbol{\beta}}\left(\prod_{i=1}^{p} \prod_{j=1}^{n} f_{i}\left(y_{i j} \mid \eta_{i j}\right)\right) \frac{1}{(2 \pi)^{n p / 2}}(\operatorname{det}(\mathbf{D}))^{-1 / 2} \times \\
& \times \exp \left\{-\frac{1}{2}(\boldsymbol{\eta}-\mathbf{X} \boldsymbol{\beta})^{\prime} \mathbf{D}^{-1}(\boldsymbol{\eta}-\mathbf{X} \boldsymbol{\beta})\right\} d \boldsymbol{\eta} \pi(\boldsymbol{H}) d \boldsymbol{H} \frac{1}{(\operatorname{det}(\boldsymbol{\Gamma}))^{(p+1) / 2}} d \boldsymbol{\Gamma} d \boldsymbol{\beta}
\end{aligned}
$$

We make a change of variable from $\boldsymbol{\eta} \rightarrow \boldsymbol{\epsilon}$ using the transformation $\boldsymbol{\epsilon}=\boldsymbol{\eta}-\boldsymbol{X} \boldsymbol{\beta}$. Next, write $\operatorname{det}(\boldsymbol{D})=\operatorname{det}(\boldsymbol{\Gamma})^{n} \times g_{0}(\boldsymbol{H})$ for some function $g_{0}$ of $\boldsymbol{H}$, and note that the expression within the exponent can be simplified to $-\frac{1}{2} \operatorname{tr}\left(\boldsymbol{\Gamma}^{-1} \boldsymbol{S}\right)$ where

$$
\boldsymbol{S}=\sum_{j, l=1}^{n} \boldsymbol{H}_{j l} \boldsymbol{\epsilon}_{j} \boldsymbol{\epsilon}_{l}^{\prime}
$$

with $\boldsymbol{H}_{j l} \equiv-w_{j l} \boldsymbol{H}$ if $j \neq l$, and $\boldsymbol{H}_{j j}=w_{j+} \boldsymbol{I}_{p}$. Now, integrating with respect to $\boldsymbol{\Gamma}$, the marginal reduces to

$$
\boldsymbol{m}(\boldsymbol{y})=\int_{\boldsymbol{H}} \int_{\boldsymbol{\beta}} \int_{\boldsymbol{\epsilon}}\left(\prod_{i=1}^{p} \prod_{j=1}^{n} f_{i}\left(y_{i j} \mid \epsilon_{i j}+x_{i j}^{\prime} \beta_{i}\right)\right) \frac{1}{(\operatorname{det}(\boldsymbol{S}))^{n / 2}} d \boldsymbol{\epsilon} d \boldsymbol{\beta}\left(g_{0}(\boldsymbol{H})\right)^{-1} \pi(\boldsymbol{H}) d \boldsymbol{H},
$$

ignoring proportionality constants. We make a change of variable from $\boldsymbol{\epsilon} \rightarrow \boldsymbol{u}$ defined by $\epsilon_{11}=u_{11}$ and $\epsilon_{i j}=u_{i j} u_{11}$ for $(i, j) \neq(1,1)$ with an associated Jacobian of $u_{11}^{n p-1}$. With this substitution,

$$
\boldsymbol{S}=u_{11}^{2} \boldsymbol{S}^{*}
$$

where $\boldsymbol{S}^{*}=\sum_{j, l=1}^{n} \boldsymbol{H}_{j l} \boldsymbol{U}_{j l}$ where $\boldsymbol{U}_{j l}=V_{j} V_{l}^{\prime}$ with $V_{1}=\left(1, u_{21}, u_{31}, \cdots, u_{p 1}\right)^{\prime}$ and $V_{j}=$ $\left(u_{1 j}, u_{2 j}, \cdots, u_{p j}\right)$ for $j \geq 2$. It follows that

$$
\operatorname{det}(\boldsymbol{S})=u_{11}^{2 p} \operatorname{det}\left(\boldsymbol{S}^{*}\right)
$$

Subsequently,

$\boldsymbol{m}(\boldsymbol{y})=\int_{\boldsymbol{H}} \int_{\boldsymbol{\beta}} \int_{\boldsymbol{u}}\left(\prod_{i=1}^{p} \prod_{j=1}^{n} f_{i}\left(y_{i j} \mid u_{i j}^{*} u_{11}+x_{i j}^{\prime} \beta_{i}\right)\right) \frac{1}{u_{11}} \frac{1}{\left(\operatorname{det}\left(\boldsymbol{S}^{*}\right)^{n / 2}\right)} d \boldsymbol{u} d \boldsymbol{\beta}\left(g_{0}(\boldsymbol{H})\right)^{-1} \pi(\boldsymbol{H}) d \boldsymbol{H}$,

where $u_{i j}^{*}=u_{i j}$ for $(i, j) \neq(1,1), u_{11}^{*}=1$ and $\left.\boldsymbol{S}^{*}=\sum_{j, l=1}^{n} \boldsymbol{H}_{j l} \boldsymbol{U}_{j l}\right)$. It follows that the integral with respect to $u_{11}$ diverges around $u_{11}=0$ proving that $\boldsymbol{m}(\boldsymbol{y})=\infty$.

Proof of Theorem 2: Without loss of generality, we take the first $q_{i}$ rows of $\tilde{\boldsymbol{X}}_{\mathcal{C}_{i}}$ to be the linearly independent rows. It follows that the marginal of $\boldsymbol{y}$,

$\boldsymbol{m}(\boldsymbol{y}) \leq C_{0} \int_{\boldsymbol{F}} \int_{\boldsymbol{\Gamma}} \int_{\boldsymbol{\beta}} \int_{\boldsymbol{\eta}}\left(\prod_{i=1}^{p} \prod_{j=1}^{q_{i}} f_{i j}\left(y_{i j} \mid \epsilon_{i j}+x_{i j}^{\prime} \beta_{i}\right)\right) f_{0}(\boldsymbol{\eta} \mid \boldsymbol{\beta} \boldsymbol{F}, \boldsymbol{\Gamma}) d \boldsymbol{\beta} d \boldsymbol{\eta} \pi(\boldsymbol{F}) d \boldsymbol{F} \pi_{U V}(\boldsymbol{\Gamma}) d \boldsymbol{\Gamma}$ 
where $C_{0}$ is a constant depending on $A$ and $B$ and the submatrix $X_{i}^{*}=\left(x_{i 1}^{\prime}, x_{i 2}^{\prime}, \cdots, x_{i q_{i}}^{\prime}\right)^{\prime}$ is of dimension $q_{i} \times q_{i}$ with full rank $q_{i}$. Making a change of variable from $\beta_{i} \rightarrow r_{i}=$ $\left(\epsilon_{i 1}, \epsilon_{i 2}, \cdots, \epsilon_{i q_{i}}\right)^{\prime}+X_{i}^{*} \beta_{i}$ for $i=1,2, \cdots, p$, condition (25) implies that

$$
\begin{aligned}
\boldsymbol{m}(\boldsymbol{y}) & \leq \prod_{i=1}^{p} \operatorname{det}\left(X_{i}^{*}\right) \int_{\boldsymbol{\epsilon}} \int_{\boldsymbol{F}} \int_{\boldsymbol{\Gamma}} f_{0}(\boldsymbol{\epsilon} \mid \boldsymbol{F}, \boldsymbol{\Gamma}) d \boldsymbol{\epsilon} \pi(\boldsymbol{F}) d \boldsymbol{F} \pi_{U V}(\boldsymbol{\Gamma}) d \boldsymbol{\Gamma} \\
& \leq \prod_{i=1}^{p} \operatorname{det}\left(X_{i}^{*}\right)<\infty
\end{aligned}
$$

since the integrands all integrate to finite numbers; $f_{0}$ integrates to 1 with respect to $\boldsymbol{\epsilon}$, and $\pi(\boldsymbol{F})$ and $\pi_{U V}(\boldsymbol{\Gamma})$, respectively integrates to 1 with respect to $\boldsymbol{F}$ and $\boldsymbol{\Gamma}$ since they are proper priors.

Derivation of the Jacobian from $(\boldsymbol{\Gamma}, \boldsymbol{Q}) \rightarrow \boldsymbol{B}$ : We consider three transformation steps: $(\boldsymbol{\Gamma}, \boldsymbol{Q}) \stackrel{(a)}{\rightarrow}\left(\boldsymbol{\Gamma}^{-\mathbf{1}}, \boldsymbol{Q}\right) \stackrel{(b)}{\rightarrow}(\boldsymbol{L}, \boldsymbol{Q}) \stackrel{(c)}{\rightarrow} \boldsymbol{B}$, where $(a)$ is an inverse transformation, $(b)$ is Cholesky decomposition and $(c)$ is QR decomposition. The Jacobian of each transformation can be obtained as follows (Muirhead (1982)): (a) $d \boldsymbol{\Gamma}=\operatorname{det}\left(\boldsymbol{\Gamma}^{-\mathbf{1}}\right)^{-(p+1)} d \boldsymbol{\Gamma}^{-1}$, (b) $d \boldsymbol{\Gamma}^{-\mathbf{1}}=2^{p} \prod_{i=1}^{p} L_{i i}^{p+1-i} d \boldsymbol{L}^{\prime}$, and $(\mathrm{c}) d \boldsymbol{B}=\prod_{i=1}^{p} L_{i i}^{p-i} d \boldsymbol{L}^{\prime}\left(\boldsymbol{Q} d^{*} \boldsymbol{Q}^{\prime}\right)$, where $\left(\boldsymbol{Q} d^{*} \boldsymbol{Q}^{\prime}\right)$ defines the Haar measure on the set of $p \times p$ orthogonal matrices. Thus, defining $d \boldsymbol{Q} \propto\left(\boldsymbol{Q} d^{*} \boldsymbol{Q}^{\prime}\right)$, we have $d \boldsymbol{\Gamma} d \boldsymbol{Q}=\operatorname{det}\left(\boldsymbol{\Gamma}^{-\mathbf{1}}\right)^{-(p+1)} 2^{p} \prod_{i=1}^{p} L_{i i}^{p+1-i} d \boldsymbol{L}^{\prime} d \boldsymbol{Q} \propto \operatorname{det}\left(\boldsymbol{\Gamma}^{-\mathbf{1}}\right)^{-(p+1)} \prod_{i=1}^{p} L_{i i} d \boldsymbol{B} \propto$ $\operatorname{det}\left(\boldsymbol{\Gamma}^{-\mathbf{1}}\right)^{-p-1 / 2} d \boldsymbol{B} \propto \operatorname{det}\left(\boldsymbol{B} \boldsymbol{B}^{\prime}\right)^{-(p+1 / 2)} d \boldsymbol{B}$. 


\begin{tabular}{|c|ccc|ccc|ccc|ccc|}
\hline$\theta$ & \multicolumn{3}{|c|}{$\boldsymbol{\beta}$} & \multicolumn{3}{c|}{$\boldsymbol{\Gamma}$} & \multicolumn{3}{c|}{$\boldsymbol{F}$} & \multicolumn{3}{c|}{$\boldsymbol{\eta}$} \\
\hline Prior & (I) & $(\mathrm{II})$ & $(\mathrm{III})$ & $(\mathrm{I})$ & $(\mathrm{II})$ & $(\mathrm{III})$ & $(\mathrm{I})$ & $(\mathrm{II})$ & $(\mathrm{III})$ & $(\mathrm{I})$ & $(\mathrm{II})$ & $(\mathrm{III})$ \\
\hline RMAD & 0.253 & 0.250 & 0.258 & 0.799 & 1.660 & 2.994 & 0.956 & 0.982 & 0.990 & 0.557 & 0.533 & 0.558 \\
MSE & 0.017 & 0.017 & 0.017 & 0.045 & 0.113 & 0.336 & 0.166 & 0.167 & 0.169 & 0.066 & 0.071 & 0.085 \\
CP & 0.861 & 0.894 & 0.925 & 0.896 & 0.618 & 0.000 & 0.881 & 0.859 & 0.859 & 0.874 & 0.933 & 0.932 \\
W & 0.383 & 0.430 & 0.478 & 0.546 & 0.642 & 0.027 & 1.236 & 1.161 & 1.107 & 0.787 & 0.943 & 1.040 \\
\hline
\end{tabular}

Table 1: Deviations measures for averages over $\boldsymbol{\beta}, \boldsymbol{\Gamma}, \boldsymbol{F}$ and $\boldsymbol{\eta}$ for the complete data case.

\begin{tabular}{|c|ccc|ccc|ccc|ccc|}
\hline$\theta$ & \multicolumn{3}{|c|}{$\boldsymbol{\beta}$} & \multicolumn{3}{c|}{$\boldsymbol{\Gamma}$} & \multicolumn{3}{c|}{$\boldsymbol{F}$} & \multicolumn{3}{c|}{$\boldsymbol{\eta}$} \\
\hline Prior & (I) & $(\mathrm{II})$ & $(\mathrm{III})$ & $(\mathrm{I})$ & $(\mathrm{II})$ & $(\mathrm{III})$ & $(\mathrm{I})$ & $(\mathrm{II})$ & $(\mathrm{III})$ & $(\mathrm{I})$ & $(\mathrm{II})$ & $(\mathrm{III})$ \\
\hline RMAD & 0.256 & 0.254 & 0.263 & 1.037 & 1.782 & 2.992 & 0.916 & 0.928 & 0.929 & 0.539 & 0.559 & 1.132 \\
MSE & 0.018 & 0.018 & 0.018 & 0.074 & 0.143 & 0.336 & 0.155 & 0.157 & 0.158 & 0.071 & 0.075 & 0.090 \\
CP & 0.911 & 0.936 & 0.951 & 0.923 & 0.634 & 0.000 & 0.931 & 0.890 & 0.882 & 0.889 & 0.936 & 0.934 \\
W & 0.445 & 0.495 & 0.534 & 0.710 & 0.746 & 0.026 & 1.294 & 1.210 & 1.157 & 0.840 & 0.977 & 1.059 \\
\hline
\end{tabular}

Table 2: Deviations measures for averages over $\boldsymbol{\beta}, \boldsymbol{\Gamma}, \boldsymbol{F}$ and $\boldsymbol{\eta}$ for the missing data case. 


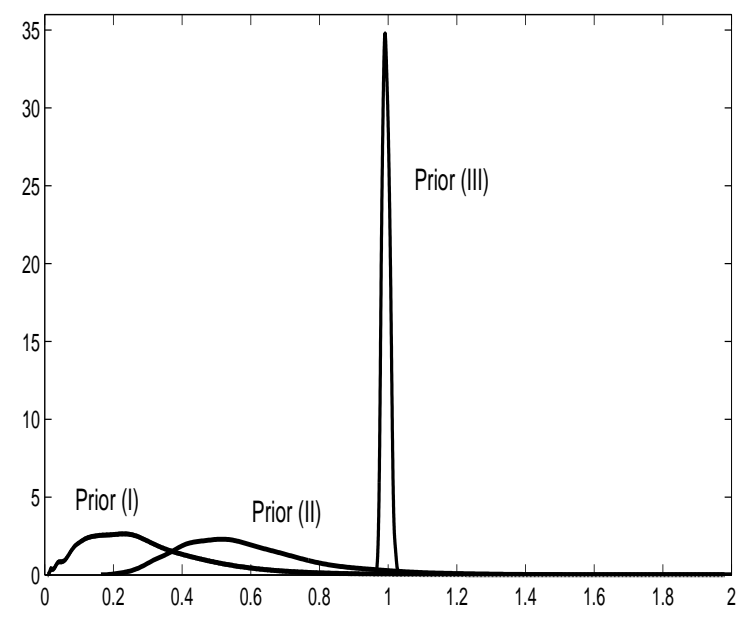

Figure 1: Posterior densities for $\Gamma_{22}$ corresponding to the three prior choices. 
Figure 2: Observed SMR of Lung Cancer and Poverty in Michigan

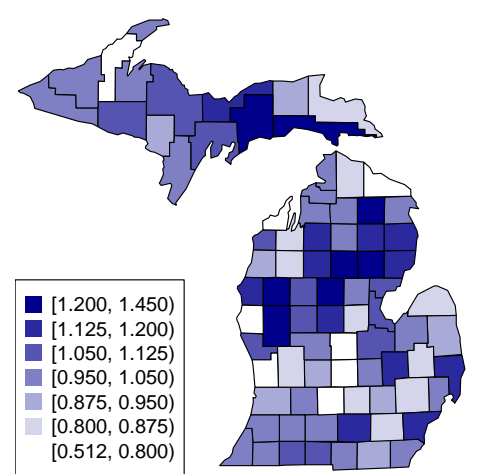

(a) Obs SMR

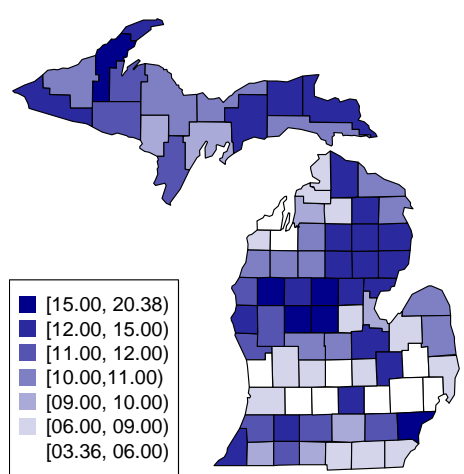

(b) Poverty $(\%)$ 